\title{
Pacientes asistidos en el Departamento de Medicina de un hospital pediátrico en el inicio de la pandemia COVID-19 en Buenos Aires, Argentina
}

\author{
Patients assisted at the Department of Medicine of a pediatric hospital \\ at the beginning of the COVID-19 pandemic in Buenos Aires, Argentina
}

\author{
Dr. Héctor Cairoli ${ }^{a}$ Dra. Silvina Raiden ${ }^{a}$ Dra. María J. Chiolo ${ }^{b}$ Dra. Sandra Di Lalla y Dr. Fernando Ferrero ${ }^{a}$ \\ Colaboradores: Dr. José Blanco Vitorero, Dr. Edgardo Checacci, Dra. Carolina Davenport, Dra. Paula Domínguez, \\ Dr. Horacio Planovsky, Dr. Fabián Gambarruta, Dr. Mariano Ibarra, Dra. María Fabiana Ossorio, \\ Dr. Javier Potasnik, Dra. Norma Schenone, Dra. Milagros Torrents y Dr. Fernando Torres.
}

\section{RESUMEN}

Introducción. A pesar de la magnitud de la pandemia por COVID-19, la información sobre su desarrollo en pediatría es aún limitada. Se describen las características de pacientes hospitalizados por COVID-19 en un hospital pediátrico durante los primeros tres meses de la pandemia.

Método. Estudio descriptivo, que incluyó a todos los pacientes hospitalizados por COVID-19, desdeel 1/4 / 2020 al 30 / 6 / 2020. Resultados. Se hospitalizaron 191 pacientes por COVID-19; la edad mediana fue 7,7 años; el $89 \%$ presentaban antecedente de contacto. El 35,6\% se consideraron asintomáticos; el 61,2\%, leves, y el3,2\%, moderados (no se observaron pacientes graves). Ninguno recibió tratamiento específico para la enfermedad. Los síntomas más frecuentes fueron fiebre, odinofagia y tos. La duración de la hospitalización tuvo una mediana de 6 días. Conclusión. Se reportaron 191 casos de niños y adolescentes hospitalizados por COVID-19. La mayoría fueron asintomáticos o presentaron enfermedad leve.

Palabras clave: infecciones por coronavirus, COVID-19, neumonía.

http:/ / dx.doi.org/10.5546/ aap.2020.423

Texto completo en inglés:

http:/ / dx.doi.org/10.5546/ aap.2020.eng.423

Cómo citar: Cairoli H, Raiden S, Chiolo MJ, Di Lalla S, Ferrero F. Pacientes asistidos en el Departamento de Medicina de un hospital pediátrico en el inicio de la pandemia COVID-19 en Buenos Aires, Argentina. Arch Argent Pediatr 2020;118(6):423-426.

a. Departamento de Medicina.

b. Departamento de Cirugía.

c. Departamento de Consultorios Externos.

Hospital General de Niños Pedro de Elizalde,

Ciudad Autónoma de Buenos Aires, Argentina.

Correspondencia:

Dr. Fernando Ferrero: fferrero@intramed.net

Financiamiento: Ninguno.

Conflicto de intereses: Ninguno que declarar.

Recibido: 7-7-2020

Aceptado: 13-8-2020

\section{INTRODUCCIÓN}

En pocos meses, la enfermedad ahora conocida como COVID-19 alcanzó una distribución global y generó millones de casos y centenas de miles de muertos en todo el mundo. ${ }^{1}$ Aunque la información aún es limitada, esta pandemia parecería ser menos grave en la población pediátrica, pues la mayoría de los casos se describen con pocos síntomas, buena evolución y una muy baja morbimortalidad. ${ }^{2}$

Un reporte chino precoz refiere que solo el $5,9 \%$ de los casos pediátricos fueron considerados graves. ${ }^{3}$ Información posterior proveniente de Europa parece confirmar esta estimación inicial. ${ }^{4}$

Dado que el desarrollo de la pandemia parece tener alguna relación con variables socioeconómicas-culturales, la forma en que se expresa en cada país podría tener características particulares. ${ }^{5}$ En la Argentina, aún hay poca información sobre el desarrollo de la pandemia en pediatría. El objetivo del presente trabajo es describir las características de los pacientes hospitalizados en relación con la pandemia COVID-19 en un hospital pediátrico durante sus primeros tres meses de desarrollo.

\section{POBLACIÓN Y MÉTODOS}

Diseño: Estudio descriptivo.

Población: Pacientes hospitalizados en relación con la pandemia COVID-19 en áreas específicas del Hospital General de Niños Pedro de Elizalde destinadas a tal fin, desde el 1/4/ 2020 al 30/6/2020.

Esta población incluyó a pacientes con COVID-19 pesquisados en el Departamento de Urgencias del Hospital, derivados desde diferentes efectores de salud con diagnóstico ya confirmado, y casos sospechosos que estaban esperando el resultado de la pesquisa. También 
se incluyeron pacientes con otras patologías que requerían internación a los que se consideró sospechosos, ya fuera antes del ingreso o durante su estancia en el Hospital.

El sector destinado a COVID-19 incluyó un área para sospechosos con estricto aislamiento individual y dos áreas para pacientes confirmados con capacidad para agrupar en cohortes y asistirlos integralmente como consecuencia de esta enfermedad y/o patología de base.

Variables en estudio: En todos los casos, se registró el resultado de la pesquisa de coronavirus tipo 2 del síndrome respiratorio agudo grave (SARS-CoV-2), mediante reacción en cadena de la polimerasa en tiempo real (real time polymerase chain reaction; $R T-P C R$, por sus siglas en inglés), sexo, edad, lugar de residencia, antecedente epidemiológico (contacto estrecho con un infectado), presencia de comorbilidad y duración de la hospitalización. La gravedad se evaluó según lo descrito por Dong. ${ }^{3}$

Procesamiento estadístico: Se describieron las variables categóricas usando porcentajes con intervalos de confianza del $95 \%$ (IC $95 \%$ ) $\mathrm{y}$, para las variables continuas, mediana e intervalo intercuartílico (IIC), según el ajuste a la normalidad (Kolmogorov-Smirnov). El procesamiento de datos se efectuó con IBM SSPS Statistics 20.0.

Consideraciones éticas: Se solicitó y obtuvo autorización del Comité de Ética en Investigación y la Dirección de la Institución.

\section{RESULTADOS}

En el período estudiado, se hospitalizaron 601 niños y adolescentes en el área destinada a COVID-19, acompañados por 516 adultos responsables. El número de hospitalizados por semana varió entre 11 y 84 (Figura 1).

En el $31,8 \%$ de los niños y el $28,5 \%$ de los acompañantes, se confirmó la presencia de $S A R S-C o V-2$ por PCR. De los pacientes en los que se descartó la enfermedad $(\mathrm{n}=410)$, el $80,7 \%$ fue externado oportunamente, y el 19,3\%, trasladado a otro sector (no COVID) del Hospital por requerirlo debido a su enfermedad (por ejemplo, pacientes con patología oncológica o quirúrgica).

En los 191 pacientes con COVID-19, la edad mediana fue 7,7 años (IIC: 1,7-13,1). El 53,9\% eran varones. El 85,3\% tenían su residencia dentro de la jurisdicción de la Institución (Ciudad Autónoma de Buenos Aires-CABA-) y, de ellos, el 41,1\% vivían en un barrio considerado vulnerable. El $89 \%$ tenían antecedente epidemiológico. El 27,7\% presentaba comorbilidades; el asma fue la más frecuente (el 5,8\%) (Tabla 1).

En relación con la presentación clínica, el $35,6 \%$ fueron asintomáticos; el 61,2 \%, leves, y el 3,2 \%, moderados. No se observaron pacientes graves ni críticos. ${ }^{8}$ Ningún paciente recibió tratamiento específico para la enfermedad. El síntoma inicial predominante más frecuente fue fiebre, seguido por odinofagia y tos (Tabla 2).

La duración de la hospitalización tuvo una

FIGURA 1. Hospitalizaciones relacionadas con COVID-19 según la semana epidemiológica y los resultados de la reacción en cadena de la polimerasa para SARS-CoV-2

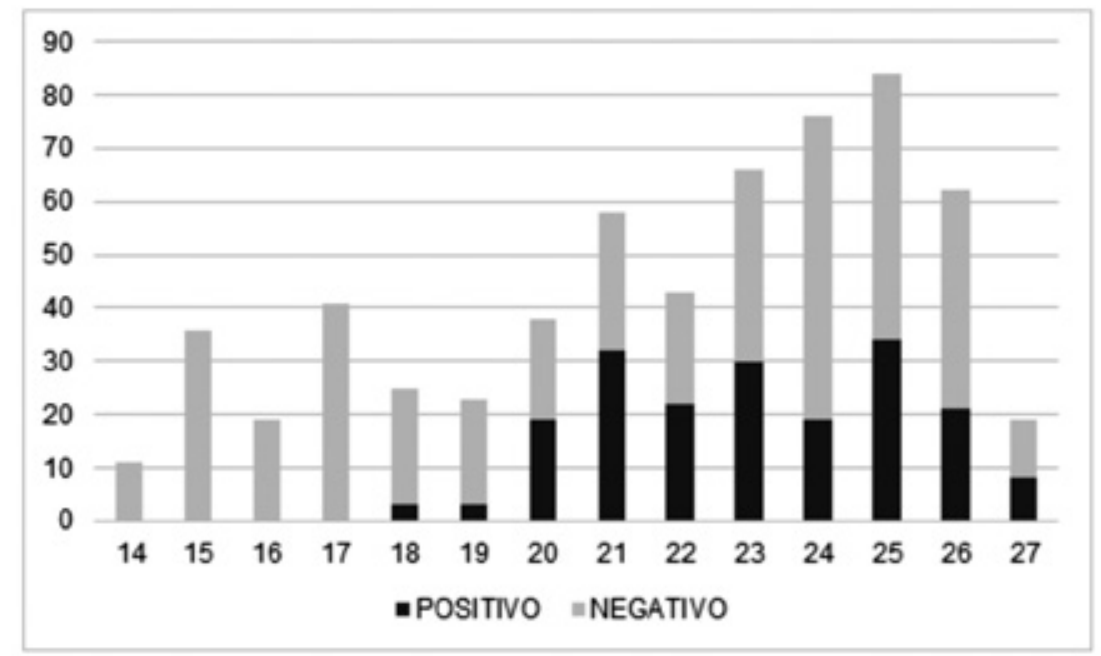

(La semana 27, que finaliza el 5/7/2020, solo incluye los casos ingresados hasta el 30/6/2020). 
mediana de 6 días (IIC: 4-9). Al momento de este reporte, los pacientes con COVID-19 habían sido dados de alta sin complicaciones (con seguimiento telefónico) o permanecían hospitalizados para su tratamiento, cuando su enfermedad de base lo requería.

\section{DISCUSIÓN}

En este estudio, se reporta nuestra experiencia inicial en la asistencia de pacientes pediátricos con COVID-19. En los tres primeros meses de la pandemia en nuestro país, se asistieron 191 pacientes.

En ese mismo período, la CABA reportó 32806 casos, 4719 (el 14,4\%) de los cuales fueron menores de 18 años. ${ }^{6}$ Reportes de China refirieron que el 2,1\% de los casos de COVID-19 correspondían a niños y adolescentes. ${ }^{7}$ En Italia, se mencionó que el 1,2\% de los casos ocurrieron en este grupo etario ${ }^{8} y$, en EE. UU., hasta abril, se había reportado que el 1,7\% de los casos correspondían a niños y adolescentes. ${ }^{9}$ Aunque es un punto todavía en discusión, es posible que la conformación etaria y los hábitos sociales de las diferentes poblaciones incidan en las diferencias observadas. ${ }^{10}$ No debe olvidarse que, por ejemplo, la Argentina tiene casi el doble de población menor de 15 años que Italia (según datos del Banco Mundial, el $25 \%$ vs. el $13 \%$ ).

También existen diferencias en la proporción de niños y adolescentes que requirieron hospitalización. Un estudio multicéntrico europeo que incluyó a 582 niños con infección por $S A R S-C o V-2$ reportó que el $62 \%$ requirieron hospitalización. ${ }^{11}$ Los Centers for Disease Control and Prevention (CDC) informaron una tasa de hospitalización general por COVID-19 para EE. UU. de 102,5 por cien mil habitantes, en que

TABla 1. Comorbilidades en pacientes pediátricos hospitalizados con COVID-19 $(n=26)$

\begin{tabular}{lc}
\hline Diagnóstico o antecedente & n \\
\hline Asma & 12 \\
Diabetes mellitus & 2 \\
Encefalopatía crónica & 2 \\
Meduloblastoma & 2 \\
Obesidad & 2 \\
ACV hemorrágico & 1 \\
Herpes zóster & 1 \\
Síndrome convulsivo & 1 \\
Lesión renal crónica & 1 \\
Lupus eritematoso sistémico & 1 \\
Púrpura trombocitopénica & 1 \\
\hline
\end{tabular}

ACV: accidente cerebrovascular.
8,9 fueron de 0-4 años de edad y 4,0, entre 5 y 17 años. ${ }^{12}$

Además, en los resultados de nuestro estudio, es probable que hayan influido otros factores. Por un lado, la pesquisa sistemática en la población infantil de barrios con elevada circulación viral comunitaria, seguramente, incrementó la proporción de niños con la infección, aunque la mayoría hayan sido asintomáticos. Por otro lado, una parte sustancial de la población asistida en nuestro Hospital provenía de barrios vulnerables y carecían de las condiciones exigidas para acceder al aislamiento extrahospitalario para sujetos asintomáticos, por lo cual debieron ser hospitalizados. ${ }^{13}$ Finalmente, también pudo haber influido la decisión de nuestro Hospital de pesquisar sistemáticamente a todo paciente que requiriera hospitalización por otro motivo desde finales de mayo.

En relación con la gravedad de la enfermedad en pediatría, nuestra experiencia coincide en que la mayoría de los casos fueron leves o asintomáticos. Solo se encontraron 6 casos moderados y ninguno grave/ crítico. Dong y col., en China, reportaron el $51 \%$ de casos leves, el 38,7 \% de moderados y el 5,9\% de graves/ críticos. ${ }^{3}$ Tagarro, en España, refirió que solo el 9,7 \% de los casos de su serie fueron graves. ${ }^{4} \mathrm{El}$ estudio multicéntrico europeo de Götzinger y col., mostró que el $9 \%$ requirieron cuidados intensivos y que el $16 \%$ eran asintomáticos. ${ }^{11}$

Como se mencionó anteriormente, la comparación en este punto con nuestra serie debe ser tomada con precaución. Es probable que nuestra experiencia muestre mayor proporción de pacientes leves o asintomáticos, debido a que el Ministerio de Salud del Gobierno de la Ciudad Autónoma de Buenos Aires (MS-GCABA) adoptó

TABLa 2. Presentación clínica de los pacientes hospitalizados con COVID-19 $(n=191)$

\begin{tabular}{llcc}
\hline & Síntoma & n & $\%$ \\
\hline Asintomáticos & & 68 & 35,6 \\
Sintomáticos* & Fiebre & 55 & 27,2 \\
& Odinofagia & 23 & 12 \\
& Tos & 20 & 10,5 \\
& Cefalea & 14 & 7,3 \\
& Rinorrea & 11 & 5,7 \\
& Síntomas abdominales & 6 & 3,1 \\
& Anosmia & 5 & 2,6 \\
& Disgeusia & 2 & 1 \\
& Exantema & 1 & 0,5 \\
\hline
\end{tabular}

* Síntoma inicial (los pacientes podían presentar más de un síntoma). 
una estrategia de búsqueda activa de casos en la comunidad, por lo que varios de nuestros pacientes surgieron del estudio de contactos estrechos.

La fiebre fue el síntoma más frecuente, seguido por síntomas de la vía aérea superior y cefalea, similar a lo reportado por Götzinger y col. ${ }^{11}$ Hasta el momento de efectuar este informe, no se había registrado ningún caso que se presentara como Kawasaki-like (COVID-19 associated Multisystem Inflammatory Syndrome in Children, MIS-C); sin embargo, se había reportado que la aparición de este síndrome solía manifestarse semanas después de alcanzarse el pico de casos pediátricos, al cual parecía que aún no se había llegado. ${ }^{14} \mathrm{La}$ comorbilidad más frecuente fue el asma, similar a lo reportado. ${ }^{8}$

La progresión en el número de hospitalizaciones refleja, de alguna manera, el desarrollo de la enfermedad en la CABA. También marca con cierta claridad el momento en que el MS-GCABA pasó a buscar activamente casos en barrios vulnerables. En la semana 19, comenzaron los operativos para ese fin $y$, a partir de allí, no solo aumentaron los casos hospitalizados en nuestro Centro, sino también la proporción de pacientes con SARS-CoV-2 confirmada por PCR. ${ }^{15}$

Este estudio presenta la limitación propia de referir la casuística de una sola institución. Sin embargo, al momento de escribir este reporte, nuestro Hospital era la institución pública de salud que más pacientes pediátricos con COVID-19 había asistido en la CABA.

También es importante resaltar que, como con toda la información referida a esta pandemia, hay que ser muy cautos al interpretarla por lo novedoso de la situación. Debe esperarse el resultado de estudios epidemiológicos extensos para tener una idea más precisa de esta enfermedad.

\section{CONCLUSIÓN}

En este estudio, se reportaron 191 casos de niños y adolescentes hospitalizados por COVID-19. La mayoría fueron asintomáticos o presentaron una enfermedad leve.

\section{Agradecimientos}

- Al Departamento de Urgencias y su Unidad Febril de Urgencia (UFU) por la atención inicial de los pacientes.

- Al Laboratorio Central y a su Área de Biología Molecular por la realización de las pruebas diagnósticas desde etapas tempranas de esta pandemia.
- A los médicos residentes de pediatría, que asumieron su tarea con entusiasmo y compromiso.

- A la Dra. Valeria Aprea, coordinadora del Comité de Crisis del Hospital General de Niños Pedro de Elizalde por liderar la respuesta del Hospital frente a la coyuntura.

\section{REFERENCIAS}

1. World Health Organization, Coronavirus Disease 2019 (COVID-19)Situation Report-140(WHO, 2020). [Acceso: el 8 dejuniode2020].Disponibleen:https: / www.who.int/docs / default-source / coronaviruse / situation-reports / 20200608covid-19-sitrep-140.pdf?sfvrsn=2f310900_2.

2. Ludvigsson JF. Systematic review of COVID-19 in children shows milder cases and a better prognosis than adults. Acta Paediatr. 2020; 109(6):1088 $\square 95$.

3. Dong Y, Mo X, Hu Y, Qi X, et al. Epidemiology of COVID-19 Among Children in China. Pediatrics. 2020;145(6):e20200702.

4. Tagarro A, Epalza C, Santos M, Sanz-Santaeufemia FJ, et al. Screening and Severity of Coronavirus Disease 2019 (COVID-19) in Children in Madrid, Spain. JAMA Pediatr. 2020;e201346. [publicado en línea, 8 de abril de 2020].

5. Bong CL, Brasher C, Chikumba E, McDougall R, et al. The COVID-19 Pandemic: Effects on Low and Middle-Income Countries. Anesth Analg. 2020; 131(1):86-92.

6. Argentina. Ministerio de Salud, Gobierno de la Ciudad Autónoma de Buenos Aires. Actualización de los casos de coronavirus en la Ciudad. [Acceso: el 3 de julio de 2020]. Disponible en: https://www.buenosaires.gob. ar / coronavirus / noticias / actualizacion-de-los-casos-decoronavirus-en-la-ciudad-buenos-aires.

7. The Novel Coronavirus Pneumonia Emergency Response Epidemiology Team. TheEpidemiologicalCharacteristics of anOutbreak of 2019 NovelCoronavirus Diseases(COVID-19) - China, 2020. China CDC Weekly. 2020; 2(8):113-22.

8. Livingston E, Bucher K. Coronavirus Disease 2019 (COVID-19) in Italy. JAMA. 2020; 323(14):1335.

9. CDC COVID-19 Response3 Team. Coronavirus Disease 2019 in Children - United States, February 12-April 2, 2020. MMWR Morb Mortal Wkly Rep. 2020; 69(14):422-6.

10. Dowd JB, Andriano L, Brazel DM, Rotondi V, et al. Demographic science aids in understanding the spread and fatality rates of COVID-19. Proc Natl Acad Sci U S A. 2020; 117(18):9696-8.

11. Götzinger F, Santiago-García B, Noguera-Julián A, Lanaspa $\mathrm{M}$, et al. COVID-19 in children and adolescents in Europe: a multinational, multicentre cohort study Lancet Child Adolesc Health. 2020;S2352-4642(20)30177-2. [Publicado en línea, 25 de junio de 2020].

12. CDC. COVID View. A Weekly Surveillance Summary of U.S. COVID-19 Activity. (for Week 26, ending June 27, 2020). [Acceso: el3 de julio de 2020].Disponible en: https: / / www.cdc.gov/ coronavirus / 2019-ncov/ covid-data/pdf/ covidview-07-03-2020.pdf.

13. Argentina. Ministerio de Salud, Gobierno de la Ciudad Autónoma de Buenos Aires. Manejo frente a casos sospechosos y confirmados de coronavirus (COVID 19). Versión 32. 19 de junio de 2020:16.

14. Capone CA, Subramony A, Sweberg T, Scheider J, et al. Characteristics, Cardiac involvement, and Outcomes of Multisystem Inflammatory Disease of Childhood (MIS-C) Associated withSARS-CoV-2 Infection. JPediatr. 2020;S00223476(20)30746-0. [Publicado en línea, 14 de junio de 2020].

15. Argentina. Ministerio de Salud, Gobierno de la Ciudad Autónoma de Buenos Aires. Dispositivo DetectAr en los barrios. [Acceso: el 22 de junio de 2020]. Disponible en: https: / / www.buenosaires.gob.ar/coronavirus / dispositivo-detectar. 